\title{
Public Cloud Cost Analysis Based on Game Theory
}

\author{
Rong Chen ${ }^{1,2, *}$ \\ ${ }^{1}$ School of information science and technology, Donghua University, China; \\ ${ }^{2}$ Shanghai Customs College, China. \\ *ch30n@126.com
}

Keywords: Game Theory, Public Cloud, Cost Analysis, Nash Equilibrium.

\begin{abstract}
In a hybrid cloud computing environment, how to divide workload reasonably is a problem that every rational user needs to consider. The game model of hybrid cloud computing is constructed. The cost is also taken into consideration when the user usually considers the response time. The function which takes the response time and the cost as the variable is the utility function. By solving the Nash equilibrium method, the user's strategy behavior is analyzed, and the optimal strategy of the user is determined. Through the simulation, the influence of different response time and expense ratio on the user equilibrium strategy is given and compared.
\end{abstract}

\section{Introduction}

As a new business computing service, cloud computing service has gradually become the main way of computing applications for businesses and individual users, such as Amazon's EC2, Google's App Engine and Microsoft's Azure platform [1]. With the rise of cloud computing, cloud computing users have been steadily growing in recent years. According to the definition of the National Institute of standards and Technology (NIST), the cloud computing service mode can be divided into infrastructure, namely infrastructure as a service (IaaS), platform as a service (PaaS) and software as a service (SaaS) [2]. Since the development of cloud computing, public cloud, private cloud and hybrid cloud have appeared in cloud services. The hybrid cloud is composed of public cloud and private cloud. The public cloud platform is a computing platform that integrates its own resources to provide services for third parties. The private cloud platform is a cloud computing platform that integrates the internal resources of the enterprise to serve the enterprise itself. The hybrid cloud platform refers to the integration of public cloud and private cloud to provide services to users [3]. With the development of cloud computing technology, a hybrid cloud consisting of public cloud and private cloud is the trend of future development. In a global survey of 270 companies in the Ganter, there are $46 \%$ business decision-makers to consider extending the existing self-built cloud collaboration application scheme based on deployment plan, which shows that the hybrid cloud will become the mainstream mode for a considerable period of time.

At present, there are two types of analysis of cloud computing: performance type analysis and market type analysis [4]. Performance type analysis aims to "optimize" performance performance. For example, for response time performance, it usually does not take into account the cost. The analysis of the market type should consider the factors that cost the market. Users can reduce workload response time by providing workload to public cloud, and improve user utility level from the perspective of response time. However, at the same time, the public cloud is charged according to the consumer's consumption, which reduces the user's utility level from the user's expense perspective. When the user needs to shorten the response time of the processing task, the user is willing to pay the cost to gain a better utility level. As the response time is gradually shortened, the user's spending on the execution unit is gradually reduced. Therefore, in the case of response time and cost, the decision of the user's workload decomposition is the problem in this paper. Taking the response time and cost into consideration, the game model of the hybrid cloud workload decomposition is established. The optimal task decomposition decision of the user is given by solving the Nash equilibrium method. First of all, the relevant work is reviewed. The game model of the hybrid cloud computing work load decomposition is established. Then, the user policy equilibrium is 
analyzed to get the Nash equilibrium strategy vector. The simulation and comparison of different parameters are carried out.

\section{Related Works}

Cloud computing is a new type of computing model. The user's tasks are assigned to a hybrid cloud server and device. It builds an extra computation for private clouds that allows users to perform workloads, that is, how to offload workloads from users to both public and private clouds [5]. Based on the concerns of public cloud service reliability and security, a hybrid cloud solution is proposed, which can make full use of public cloud resources to supplement the local private cloud resources [6]. Game theory of thought modeling can well analyze each player's strategy. With the rise of cloud computing, more and more scholars begin to analyze the workload decomposition from the perspective of game theory. As non-cooperative participants share resources, the ratio between the worst possible Nash equilibrium and social optimality is used as a measure of the efficiency of the system [7].

In a hybrid cloud computing environment, the user game is a triple: $G=<N,\left(s_{i}\right),\left(\mu_{i}\right)>$. Among them, $\mathrm{N}=\{1,2, \ldots, \mathrm{n}\}$ is a set of participants, which means there are $\mathrm{n}$ users. The policy set of users $\mathrm{i}$ is denoted as $\mathrm{Si}, S_{i}=\left\{\sigma_{i}, \sigma_{i} \in[0,1]\right\} . \sigma_{i}$ represents the proportion of the total amount of tasks performed by user $\mathrm{i}$ on the public cloud computing. $1-\sigma_{i}$ represents the proportion of the total amount of tasks assigned to the private cloud by user i. $u_{i}=f\left(t_{i}, c_{i}\right)$ represents the utility of user I. It is a function of the response time $t_{i}$ of the task and the cost of $c_{i}$. The private cloud is generally deployed within the enterprise, and it does not charge the enterprise users. The time for the execution of the work is to take the higher execution time on the public cloud and the private cloud. Other users commit workload to private cloud execution. The execution time on private cloud should be the execution time of all user tasks on private cloud, which can be expressed as: $t_{i}=\left\{\begin{array}{l}\frac{\left(1-\sigma_{i}\right) \omega}{\mu_{L}}+\sum_{k \neq i} \frac{\left(1-\sigma_{k}\right) \omega}{\mu_{L}}, \sigma_{i} \in[0,1] \\ 0, \sigma_{i}=1\end{array}\right.$. For the user, the cost will increase and the utility will become smaller as the proportion of the workload is increased. However, time decreases gradually, making utility more effective as time decreases. Thus, $\frac{\partial u_{i}}{\partial c_{i}}<0, \frac{\partial u_{i}}{\partial t_{i}}<0$.

\section{Experiment}

\subsection{Equilibrium Analysis}

The utility of each user is related to the policy of other users to submit to the public cloud. If each user is regarded as a rational participant, the policy of the user $i$ is $\sigma_{i}$. Both $t_{i}$ and $c_{i}$ are a function of $\sigma_{i}$. The utility of user $i$ can be expressed as $u_{i}\left(\sigma_{i}, \sigma_{-i}\right)$, that is, $f\left(t_{i}, c_{i}\right)=u_{i}\left(\sigma_{i}, \sigma_{-i}\right)$. The range of user policies is $\sigma_{i} \in[0,1]$. Since all users act simultaneously, this game is a complete information static game. The utility of the user is $u_{1}\left(\sigma_{1}, \sigma_{-1}\right), \ldots u_{i}\left(\sigma_{i}, \sigma_{-i}\right), \ldots, u_{n}\left(\sigma_{n}, \sigma_{-n}\right)$. With the increase of $\sigma_{i}$, the marginal utility of the user decreases. $u_{i}\left(\sigma_{i}, \sigma_{-i}\right)$ is a concave function, that is, $\frac{\partial u_{i}^{2}\left(\sigma_{i}, \sigma_{-i}\right)}{\partial \sigma_{i}^{2}}<0, \frac{\partial u_{i}^{2}\left(\sigma_{i}, \sigma_{-i}\right)}{\partial \sigma_{i} \partial \sigma_{j}}<0, \forall i, j \in N$.

The way to find Nash equilibrium is: by first order partial derivative of the payment function and its result equal to zero, $\frac{\partial u_{i}\left(\sigma_{i}, \sigma_{-i}\right)}{\partial \sigma_{i}}=0, i \in N$, the reaction function $\sigma_{i}=B\left(\sigma_{-i}\right), i \in N$ is obtained. 
That is, $\mathrm{n}$ reaction functions are obtained. The intersection of these reaction functions is Nash equilibrium. $\sigma_{i}^{*}=B_{i}\left(\sigma_{-i}\right), \forall i \in N, B_{i}\left(\sigma_{-i}\right)=\left\{\sigma_{i} \in S_{i}: u_{i}\left(\sigma_{i}, \sigma_{-i}\right)\right\} \geq u_{i}\left(\sigma_{i}^{\prime}, \sigma_{-i}\right) \forall \sigma_{i}^{\prime} \in S_{i}$.

Suppose $\frac{\partial u_{1}\left(\sigma_{1}, \sigma_{-1}\right)}{\partial \sigma_{1}}=0, \ldots, \frac{\partial u_{i}\left(\sigma_{i}, \sigma_{-i}\right)}{\partial \sigma_{i}}=0, \ldots, \frac{\partial u_{n}\left(\sigma_{n}, \sigma_{-n}\right)}{\partial \sigma_{n}}=0$, when other users are optimal, the strategy of user $\mathrm{i}$ is also its optimal strategy, that is, they are the best response strategies to each other. $\left(\sigma_{i}^{*}, \sigma_{-i}^{*}\right)$ is the Nash equilibrium of the game.

In practice, the law of diminishing marginal utility is often taken into account. In a hybrid cloud computing environment, it is a matter of concern for every rational cloud user to divide the amount of work reasonably. The function that the user i costs is $c_{i}=p \sigma_{i} \omega$. The expression of the user $i$ to perform the workload decomposition response time is $t_{i}=\frac{\left(1-\sigma_{i}\right) \omega}{\mu_{L}}+\sum_{k \neq i} \frac{\left(1-\sigma_{k}\right) \omega}{\mu_{L}}, \sigma_{i} \in[0,1]$. Then, formula (1) can be obtained:

$$
\sum_{i=1}^{n} \sigma_{i}^{*}=\frac{n^{2}}{n+p \mu_{L}\left(\frac{\alpha p \mu_{l}}{\beta}\right)^{\frac{1}{\gamma-1}}}
$$

This is a complete static game of information at the same time, so there are:

$$
\sigma_{i}^{*}=\frac{n}{n+p \mu_{L}\left(\frac{\alpha p \mu_{l}}{\beta}\right)^{\frac{1}{\gamma-1}}}
$$

$\left(\sigma_{i}^{*}, \sigma_{-i}^{*}\right)$ is the Nash equilibrium vector of the game.

\subsection{Simulation and Analysis}

When $u_{i}\left(\sigma_{i}^{*}, \sigma_{-i}^{*}\right)>u_{i}\left(1, \sigma_{-i}^{*}\right)$, the user's Nash equilibrium strategy is: $\sigma_{i}^{*}=\frac{n}{n+p \mu_{L}\left(\frac{\alpha p \mu_{l} \frac{1}{\beta}}{\beta}\right)^{\gamma-1}}$. It can be seen that the user's equilibrium strategy is related to the price, response time and cost of unit tasks.

Figure 1 has three curves. They represent three different scenarios for users 100, 1000 and 10000 , respectively. The abscissa is the unit flow price, which is between 0 and 10. Other parameters have been set. When $\mathrm{n}=100$, as the pricing of public cloud providers increases, the less subscribers are placed on the public cloud. When the total number of users $n$ increased to 1000 , with the change of pricing from 0 to 10 , the task of users submitted to public cloud was significantly improved. When the number of users increases, local resources can hardly meet the needs of users, and users submit more tasks to the public cloud. When the total number of users is increased to 10000 , the user equilibrium strategy almost divides all tasks into public cloud. This shows the necessity and superiority of the public cloud processing multi - user and multi - task. The workload of changing the number of hybrid cloud users is shown in Figure 1. 


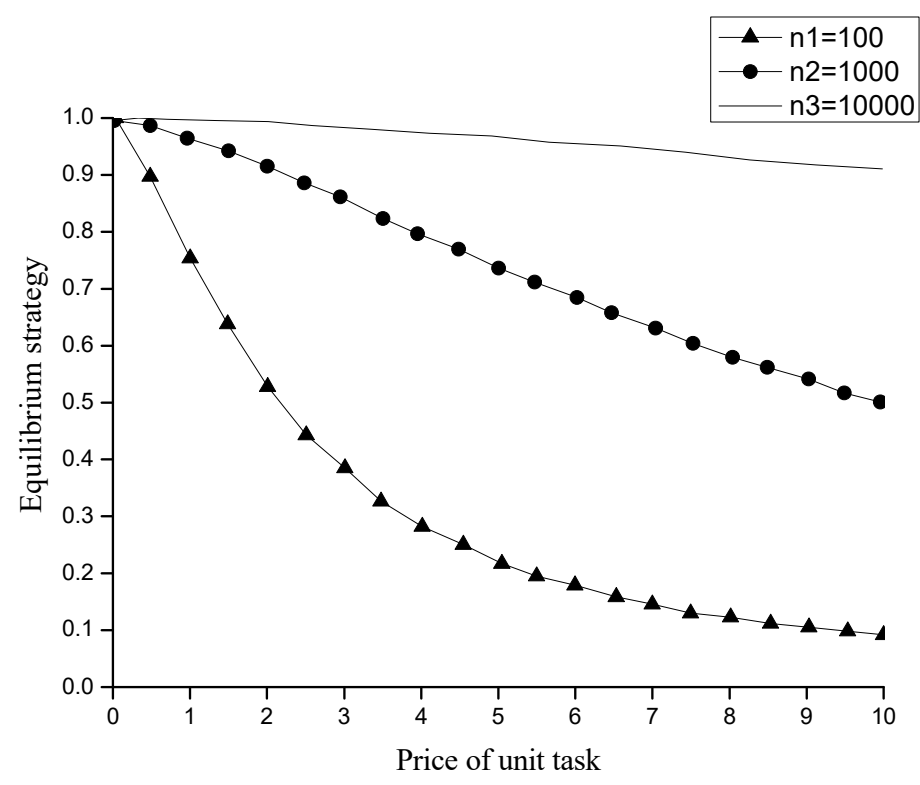

Figure 1. The workload of changing the number of hybrid cloud users

Figures 2 and 3 show the correspondence between the parameters $\alpha: \beta=10: 1$ and $\beta: \alpha=1: 10$, respectively. It shows the impact of time and cost ratio on user utility. With the change of unit task price $\mathrm{p}$, the user's equilibrium strategy under different $\alpha$ and $\beta$ also changes. As can be seen from Figure 2, the user increases with the price. Because it costs more, it reduces user utility more quickly. Users are reluctant to put more workload into the public cloud to execute. As can be seen from Figure 3 , the user's equilibrium strategy changes slowly as the price of the unit's workload changes. In other words, the cost is relatively low, making the user's utility rate is slower. The changes of time and cost is shown in Figure 2. The workload of the hybrid cloud is shown in Figure 3.

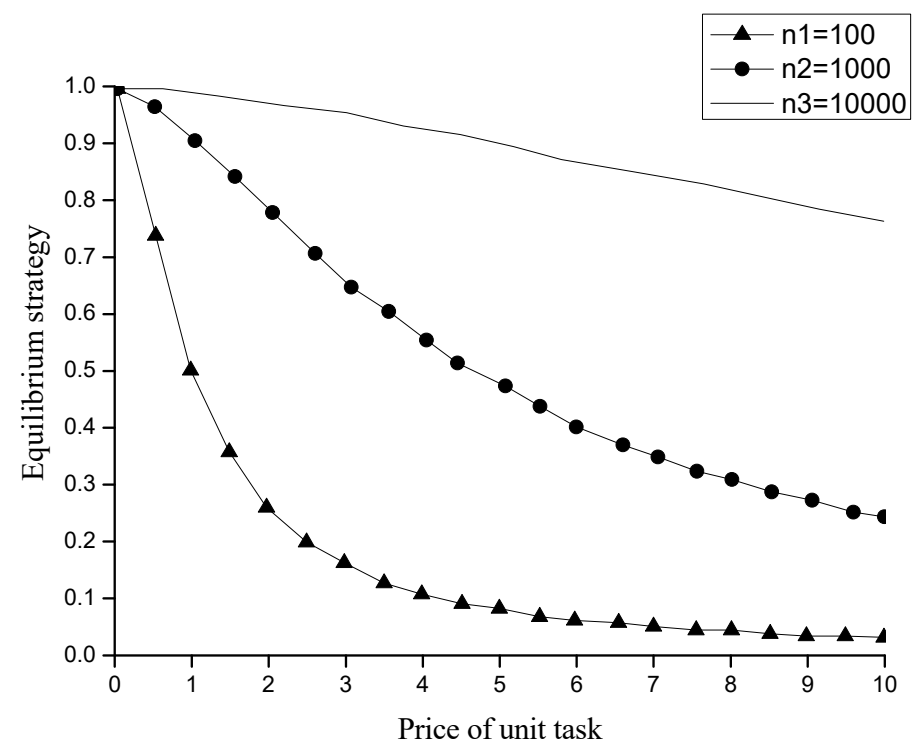

Figure 2. The changes of time and cost 


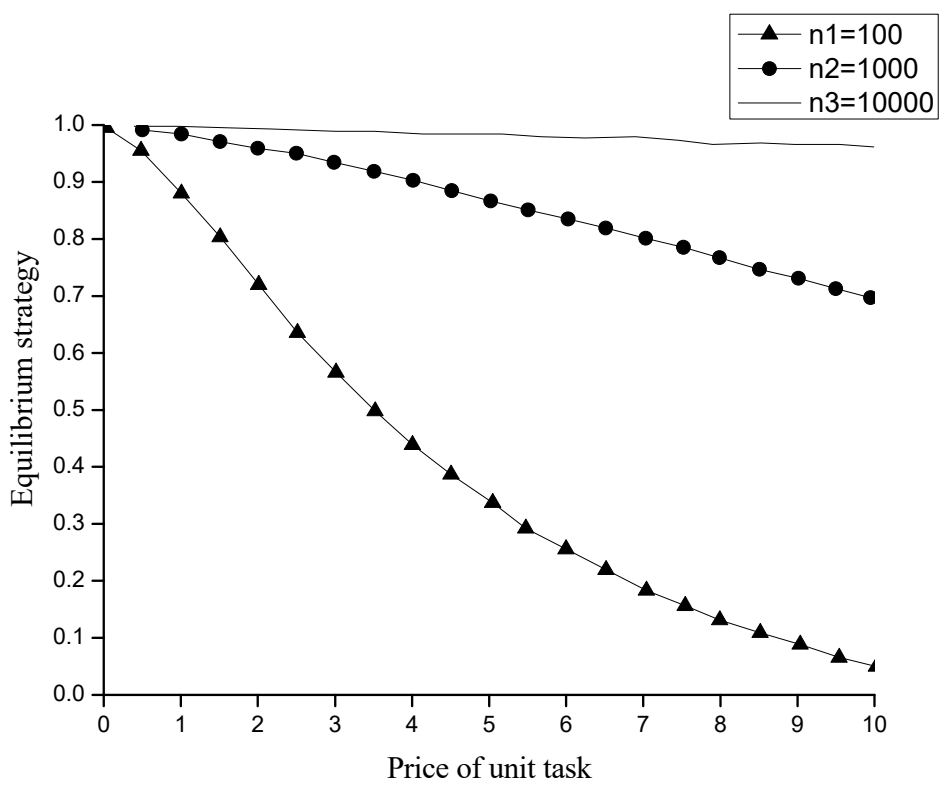

Figure 3. The workload of the hybrid cloud

\section{Conclusion}

The problem of cloud users' workload decomposition between public and private clouds in a hybrid cloud environment is proposed. Taking the response time and cost into consideration, the game model of the hybrid cloud workload decomposition is established. By solving the Nash equilibrium method, the user's optimal task decomposition decision is given. Based on the relevant research, the utility function of cost and time was selected for simulation. Under different parameters, the user's equilibrium strategy is analyzed as the price changes, which provides a theoretical basis for better decision-making for users. The analysis of workload for a given number of users is more complicated. These questions will be the direction of further research.

\section{References}

[1]. Esposito, C., Ficco, M., Palmieri, F., \& Castiglione, A. (2016). Smart cloud storage service selection based on fuzzy logic, theory of evidence and game theory. IEEE Transactions on computers, 65(8), 2348-2362.

[2]. Hu, H., Wen, Y., \& Niyato, D. (2017). Public Cloud Storage-Assisted Mobile Social Video Sharing: A Supermodular Game Approach. IEEE journal on selected areas in communications, 35(3), 545-556.

[3]. Lin, C. Y., \& Tzeng, W. G. (2017). Strategy analysis for cloud storage reliability management based on game theory. Journal of Computer Security, (Preprint), 1-19.

[4]. Chen, X., Jiao, L., Li, W., \& Fu, X. (2016). Efficient multi-user computation offloading for mobile-edge cloud computing. IEEE/ACM Transactions on Networking, 24(5), 2795-2808.

[5]. Do, C. T., Tran, N. H., Huh, E. N., Hong, C. S., Niyato, D., \& Han, Z. (2016). Dynamics of service selection and provider pricing game in heterogeneous cloud market. Journal of Network and Computer Applications, 69, 152-165.

[6]. Argoneto, P., \& Renna, P. (2016). Supporting capacity sharing in the cloud manufacturing environment based on game theory and fuzzy logic. Enterprise Information Systems, 10(2), 193210. 
[7]. Wei, W., Fan, X., Song, H., Fan, X., \& Yang, J. (2017). Imperfect information dynamic stackelberg game-based resource allocation using hidden Markov for cloud computing. IEEE Transactions on Services Computing. 